\title{
Successful Transmission Rate of Mobile Terminals with Agents in Segmented Ad Hoc Network
}

\author{
Kohei Arai \\ Department of Information Science \\ Faculty of Science and Engineering, Saga University \\ Saga, Japan
}

\author{
Lipur Sugiyanta \\ Department of Electrical Engineering \\ Faculty of Engineering, State University of Jakarta \\ Jakarta, Indonesia
}

\begin{abstract}
Mobile Wireless Ad-Hoc Network (MANET) is a special kind of network, where all of the nodes move in time. Random movement is the commonly used to model the mobility in this network. Beside as a host, each node is intended to help relaying packets of neighboring nodes using multi-hop routing mechanism in order to solve problem of dead communication. In recent years, a variety of routing protocols have been proposed specially to accommodate this environment followed with its performance evaluations, likes DSDV, DSR, AODV, and extension OSPF. Researches in this network are mostly simulation based. Research efforts haven't focused much in evaluating network performance when applied to variable number of nodes that involving agents and distributed over several network areas. The paper performed simulations using MANET simulator, which carried out based on the extension OSPF routing protocol. The modification of OSPF protocol had intended to suit the OSPF standard mechanisms for operating with high performance over wireless networks characterized by a broadcast-based transmission medium, in which the physical layer connectivity is not fully meshed. Extensive simulation scenarios have been conducted in the simulator with various numbers of nodes, random and uniform movement direction, and different agent's quantity with different size of network areas. In the performance evaluation of successful transmission (data) packets, the OSPF protocol with throughput weighted metric will be tested under different combination conditions of scenarios.
\end{abstract}

Keywords-MANET; agents; movement; packet; routing; network area.

\section{INTRODUCTION}

Wireless Ad-Hoc Network consists of mobile nodes platforms which are free to move in the area. One class of such networks has been called mobile ad hoc networks (MANETs). Node is referred to a mobile device which equipped with builtin wireless communications devices attached and has capability similar to autonomous router. The most important characteristic of MANET is the dynamic topology, which is a consequence of node mobility. Nodes can change position quite frequently, which means that we need a routing protocol that quickly adapts to topology changes. The node in an ad-hoc network can be laptops and personal digital assistants which are often very limited in resources such as CPU speed, storage capacity, battery power and bandwidth, so the routing protocol should be able to be reactive and capable of minimizing control traffic, such as periodic update messages. To be effective, the routing protocols should have keep the routing table up-to-date and reasonably small, able to choose the best route for certain destination (with weighted metrics in terms of number of hops, reliability, throughput, and cost), and capable of converged within an exchange of a small amount of messages.[8]

In MANET, mobile nodes connected with each other using multi-hop wireless links. Because there is no static infrastructure such as base stations, then if network move arbitrarily thus network topology changes frequently and unpredictably. Each node in the network forwards data packets for other nodes. It makes routing protocol design much difficult. The routing protocol which works well in fixed networks does not show the same performance in MANET. In these networks routing protocols should be more dynamic so that they quickly respond to topological changes [14]. If two nodes are not within proximity of its radio range, all packets must pass through hop by hop by one or more intermediate nodes.

To analyze MANET performance with hundreds or even thousands of nodes, the network must be split into independent layer 3 groups or domains. Smaller domains allow routing, QoS and other network protocols to operate limitary on fewer nodes, with cross-domain interaction only through overlapped area nodes. This division has several key benefits. First, it reduces overall protocol overhead. In most routing protocols, for example, the aggregate route update overhead grows as $\mathrm{O}\left(\mathrm{n}^{2}\right)$, where $\mathrm{n}$ is the number of nodes in a domain. Therefore, using smaller domains with inter-domain interaction through an overlapped area allows reducing the overall overhead. Second, it made network life time longer. Nodes have salient feature of energy-constrained devices. The battery of node is depleted by: (i) computational processing and (ii) transmission/reception of signal to maintain the signal-to-noise ratio above a certain threshold. Although the energy consumption by computations can be further reduced with new developments in low power devices, the energy consumption by communications cannot be overcome. Therefore, partitioning network into smaller domain is essential to develop efficient networking algorithms and protocols that are optimized for energy consumption. As a consequence, when partitioning a network into domains, there are some engineering rules that need to be taken into account. For example, the partition should create balanced overlapped areas; the partitions should minimize the expected traffic among different areas so as to not overload the overlapped 
area; there must be at least one path between every pair of nodes in certain area and the nodes in overlapped area, and at least one path must exist from overlapped area to nodes that belong to other area for such transmission exchange-area to be successfully take place; there must be at least one node that existed in the overlapped area to act as an relay exchange-area domain. Such nodes are called agents. Third, minimizing and managing exchange-area traffic is desirable since it helps ensuring that the backbone agents do not become congested and maximizes packet forwarding via knowledgeable exchange-area route paths. Agents ensure that no single area suffers adversely from a disproportionately large volume of overhead and data packets.

However, in this paper we focus on the impact of mobility models on the performance of data packets transmission in the large MANET that segmented into two domain areas. So our observations regarding to discuss the effect of movement mobility speed of the nodes to evaluate the performance of the improved OSPF routing protocol with weighted throughput metric, using self-made simulator that considering a dynamic network size with varying number of movement speed at an invariable pause time for mobile Ad-hoc network with frequently and fast moving nodes, movement direction of nodes and domain, either approaching agents or avoiding agents, and different size of overlapped area which potentially vary number of agents getting involved in the traffic at instance of each cycles.

The remainder of the paper is organized as follows. Section 2 gives a brief description of the related work. Section 3 discusses some limitations of the simulation model and provides the simulation model with mobility of our system and describes the effect of some metrics, Section 4 presents the evaluation performance network for data packets transmission, and finally, we conclude the paper in Section 5.

\section{RELATED WORK}

Significant academic and industrial research has led to the development of a variety of protocols, platforms and architectures for reliable communication provided by the MANET network. They are mostly centralized approaches that assume that reliable communication can be provided by the single uniform network.

Research approached in the field, like SANDMAN [13] and DEAPSpace [12], is done by grouping nodes with similar mobility patterns into clusters; in each cluster, one of the nodes (called cluster head) stays awake permanently and answers discovery requests. The rest of the nodes periodically wake up to provide the actual services and also inform the cluster head about their presence and services [1]. Other approaches take into account the network partitioning and provide related metrics and comparisons are briefly presented in the following paragraphs.

\section{A. Approach of Expanding MANET to a Large Network}

There are currently no general approaches or methodologies for the creation of domains that take into account the following important engineering constraints: balanced domains, minimal inter-domain traffic, and robust network design [3]. Most existing work on domain generation, however, has used only very limited local information. In fact, most approaches simply elect a "cluster-head" within each subnet based on node attributes like the lowest ID or highest degree [3][4]. Some proposals use local metrics during cluster generation, but the metrics are utilized just for the selection of cluster-heads [5], [6].

Steffano Galli [3] viewed the network as a weighted undirected graph $\mathrm{G}=(\mathrm{V}, \mathrm{E})$, where each vertex $\mathrm{v} \in \mathrm{V}$ represents a network node and each edge $\mathrm{e} \in \mathrm{E}$ represents a viable (potential) communication link with an associated cost we (the cost associated with every edge represents the estimated link load). An example is shown in Figure 1. We wish to partition graph $\mathrm{G}$ into $\mathrm{N}$ sub-graphs $\mathrm{G}_{\mathrm{i}}, \mathrm{i}=1,2, \ldots, \mathrm{N}$, with the objective of satisfying a given set of constraints. It is important to recognize that finding such sub-graphs, i.e. creating network partitions, requires cutting a subset $\mathrm{C} \subset \mathrm{E}$ of edges in $\mathrm{G}$. The graph $\mathrm{G}$ in Figure 1 has been partitioned in $\mathrm{N}$ $=4$ sub-graphs G1, G2, G3, and G4. The set CᄃE of cut edges is shown in dotted lines.

The rationale for choosing the partition, i.e., for deciding which edges to cut, is based on engineering rules given in the Introduction which can be translated into a graph theoretic framework as follows:

- The partition is as balanced as possible: the number of nodes in every sub-graph $G_{i}, i=1,2, \ldots, N$, is the same within the smallest possible tolerance.

- The sum of the weights of all cut edges is minimized.

- $\quad$ Each sub-graph $\mathrm{G}_{\mathrm{i}}, \mathrm{i}=1,2, \ldots, \mathrm{N}$, is 1-connected.

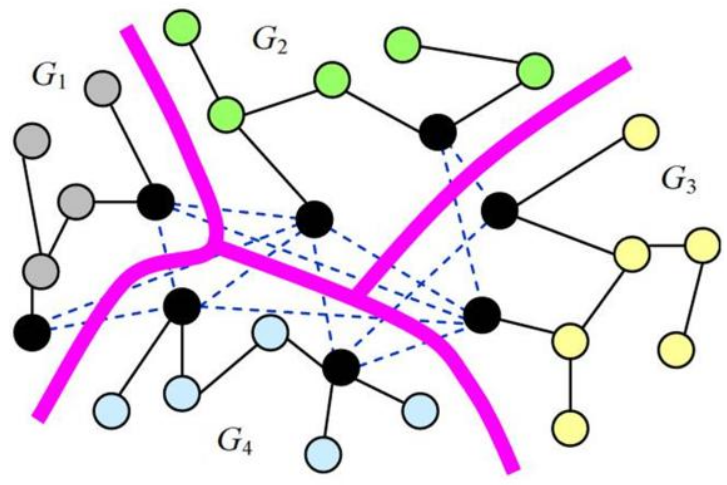

Figure 1. Example of graph partitioning (four areas) of a network [3].

Based on the Galli proposed to solve the OSPF area design problem for large networks using a two-step approach: a first step consisting of an efficient Graph Partitioning Algorithm that will create balanced areas (including a rough Area 0), and a second step consisting of additional ad-hoc heuristics that are able to ameliorate Area 0 design as well as factoring in OSPF specific metrics.

\section{B. OSPF MANET Extension}

The Open Shortest Path First routing algorithm is widely used for wired networks. As consequences of users' expectation to seamlessly coexist their mobile devices with 
their wired counterparts, the modification of legacy protocol must be designed. Due to a lack of central base station, nodes in a MANET must form peering relationships to collectively make routing decisions.

OSPF uses a link-state routing protocol to find the leastcost path from a source router to a destination router within a group of routers owned by an organization. As shown in Figure 2, a group of routers using the same routing protocol is collectively referred to as an Autonomous system (AS). Upon joining the AS, a router uses the Hello protocol to discover neighboring routers. Then it forms adjacencies with its new neighbors to exchange routing information; a process called as Link-State Advertisements (LSAs). The exchange (flooding) of routing information, allows routers to obtain complete view of the network topology. Each router stored it as link-state database (LSDB). Initially, LSDB represents the network topology that is visible by router as a result of Dijkstra leastcost path algorithm. In order to maintain and synchronize the network convergence, each router periodically broadcast updates about its current links state to their LSDBs.

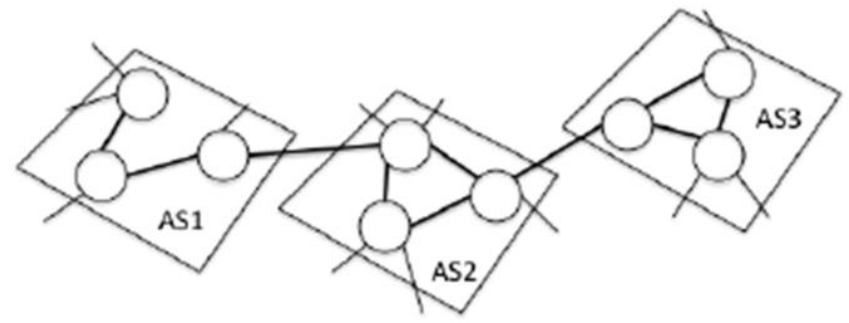

Figure 2. Connected Autonomous systems (ASs)

Although OSPF was designed for wired networks where the topology is predicable and the medium is relatively reliable, its routing algorithm can be extended to support MANET. OSPF is widely deployed and includes many innovative features. The protocol's specifications are in the public domain. It is used by upper-tier Internet Service Providers to determine routes within their networks. It supports multicast routing, multiple samecost paths, and the ability to organize a network as a hierarchy.

Nodes in a MANET randomly form peering relationships as they move along its communication range. Moreover, packet loss occurs frequently on a wireless medium due to path loss, interferences, noise, shadowing, and multipath. Therefore, OSPF needs to be modified to meet the routing requirements of MANET, which include minimizing data exchange and control overhead.

The OSPFv2 Wireless Interface Type was proposed to support wireless networks. The enhanced protocol is not limited to MANETs; it supports "wireless, broadcast-capable, multi-hop" networks. A team of Boeing Company contributed to the OSPFv2 Wireless Interface Type proposal [4]. Boeing also made other contributions to the OSPF extension for MANETs. They evaluated and analyzed the performance of MANET extensions through simulation of OSPF MANET Designated Routers (MDR) and Cisco's extension (Overlapping Relay and Smart peering) [5][6].
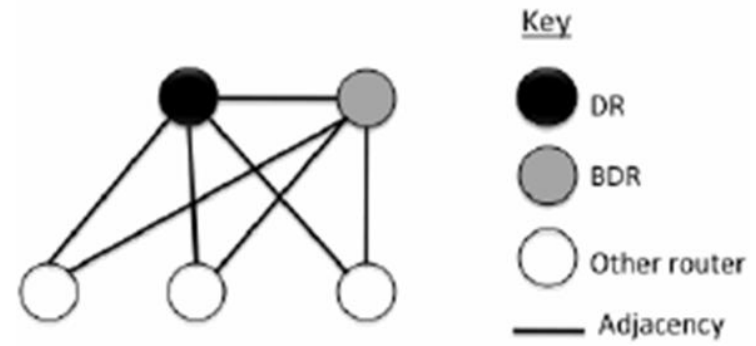

Figure 3. The router's role in the OSPF protocol to reduces the packets adjacencies.

Designated Routers (DR) algorithm provides a mechanism to reduce packets adjacency for data exchanges. The Hello protocol provides a mechanism for electing the network DR. Besides its main function to find and maintain connections among neighbors, Hello packets contain information about router priority that is used to assign the DR. The first router with the highest priority becomes the DR, and the router with the second highest, the Backup Designated Router (BDR) [15]. The DR (router) is responsible to relay the packets to other routers.

OSPF Multi-point Relaying (MPR) uses an MPR selector set to reduce adjacencies. Unlike the OSPF-MDR extension, OSPF-MPR makes not changes to Hello protocol. It minimizes flooding overhead by choosing a flooding MPR set. Similar to Cisco's extension, OSPF Multi-point Relaying (MPR) only forms adjacencies with a limited subset of neighbors to improve scalability and reduce control overhead (LSAs). However, OSPF-MPR does not assign a MDR. A router forms adjacencies with a subset of its one-hop neighbors called MPR selector set.

Most routers only form adjacencies with its MPR selector set, but some Synch routers also form adjacencies with neighbors not in their selector set. A router becomes a synch router only if it has highest router ID among its known neighbors. Hello packets and LSA contain router ID of its neighbors. At least one Synch router must exist in the MANET. However, extra data is added in Hello packets in to facilitate MPR selection.

\section{Simulation MODEL}

As foundation for this mobile environment, the core algorithm is developed from static mode (e.g., sensor networks). The enhancement algorithm for serving mobility then detailed in support of topology development, topology maintenance, and routing maintenance.

The model is initiated from broadcast mechanism and propagated through node-to-node based routing metrics approach. Each source injects single big packet which fragmented into multiple packets in the network, which traverse through the network until those reach the final destination. Packets are queued at each node in its path where it waits for an opportunity to be transmitted. This model is not only applicable in direct communication (one hop transmission) but it can also work in multi-hop transmission. 
In this situation, when the source and final destination nodes are located outside its clustered network area, source node is capable to discover multiple hop route lead to agents thus maintaining the connectivity required in comparison to standard flooding based ad hoc routing designs.

It is square of Cartesian model area with 200x200 areas and one overlapped section. We consider the case where all nodes in the network are similar, i.e., assuming a homogeneous infrastructure. Inside areas, nodes are deployed uniformly, distributed at random position in the both areas. This deployment produces a connected topology under some assumptions; sometimes a completely connected topology is built and sometimes topology is not fully connected. Simulation build a large connected component quickly using a communications radius considerably smaller than the radius needed to have the entire network connected. Agents are located in such place to facilitate communication between wireless areas and minimize the number of hops to achieve optimum throughput of mesh clients which communicate each other. Agents have static position located in the overlapped between two network areas.

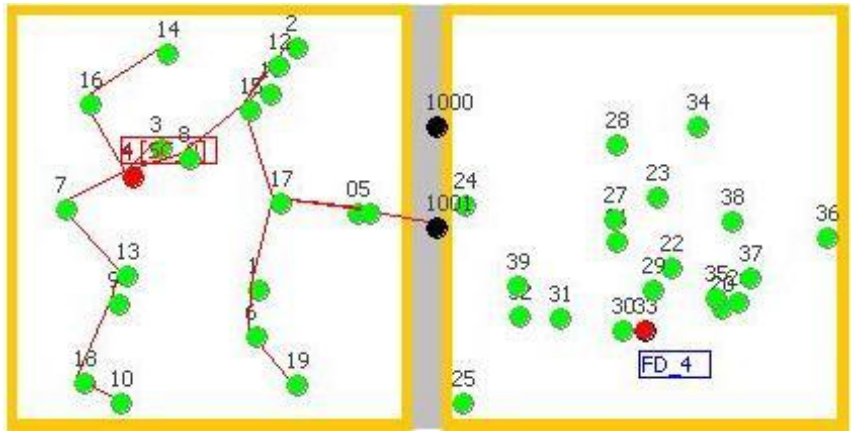

(a)

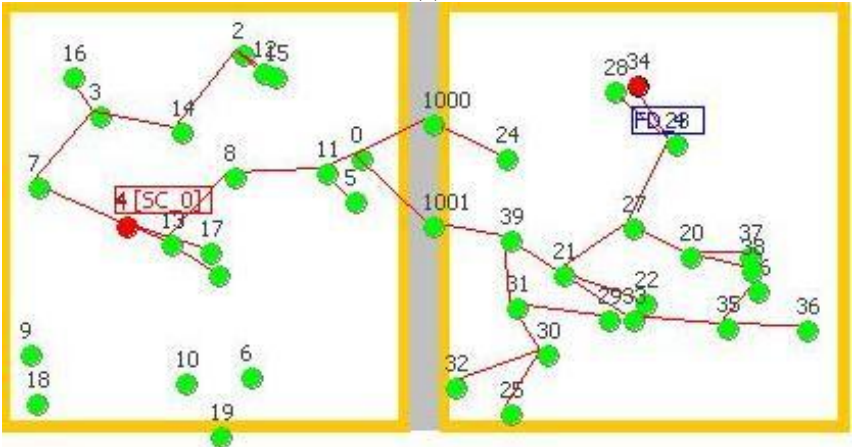

(b)

Figure 4. Development of network topology within two areas. (a) With Agents involved in delivery of packets. (b) Without Agents involved in delivery of packets.

This mode of messenger can be made clear from the Figure 4. At Figure 4(a), if the source (node 4 mark with red color) coming from certain clustered network area and having destinations (e.g. node 33) located at different (adjacent) clustered network area, the paths are able to meet at a common Agents (node 1000 or node 1001) simultaneously then only any one of the agents will be sufficient to carry all the messages to the proper destination. Thus our routing scheme utilizes the agents capability where a collection of independent request transmission come together for the purpose of cooperative task behaviors and maintaining these connectivity among pairs of sources - destinations. All these tasks essentially work directly through node - agent communication. The entire algorithm works on the fact that agents need to know the existence of each nodes at each clustered areas. On the other side, at Figure 4(b), predefined source (node 4) start the topology development protocol by sending (broadcast) an initial Hello Message. With receive-transmit subsequent routine, the process continues to all reachable nodes. Not every node will be selected to be part of the tree, and those which were not selected will keep silent (in the propagation of packet). Without any knowledge of destination topology (which is located in other network area), then the packets must travel to each vertex to reach the destination node. In both pictures, if there are more than one source nodes starts to transmit packets simultaneously, then several trees may be built in parallel.

\section{A. The Model}

Simulation consists of multi clustered network environment of homogeneous nodes that communicate with each other using the broadcast services of IEEE 802.11. There are nodes with different roles simulated in this simulation, namely initiator node/source node, receiver node, sender node, destination node, and final destination node. Initiator node/source node is node that initiates transmission of packet. Packet can be either route discovery or data transmission. Like other nodes, initiator is always moving with random direction, speed, and distance. At the time it is moving, initiator node is always sensing its neighbor to maintain connectivity. Receiver node is node that can be reached by source/sender node. Nodes are defined as neighbors if it located within its distance radius range. At initial time, node senses its neighbors before packet data is required to be transmitted. Coverage neighbor nodes always receive packets that are broadcasted from sender. Destination node is selected receiver node in multi hop transmission that should relay packets to the next receiver node. Final destination node is node that became the finish destination of packets.

The layered concept of networking was developed to accommodate changes in local layer protocol mechanism. Each layer is responsible for a different function of the network. It will pass information up and down to the next subsequent layer as data is processed. Among the seven layers in the OSI reference model, the link layer, network layer, and transport layer are 3 main layers of network. The framework is configured in those layers. Genuine packets are initiated at Protocol layer, and then delivered sequentially to next layer as assumed that fragmented packets to be randomly distributed. Simulation models each layer owned with finite buffers. Limited buffer makes packets are queued up according to the drop tail queuing principle. When a node has packets to transmit, they are queued up provide the queue contains less than $K$ elements $(K \geq 1)$. To increase the randomization of the simulation process, simulation introduces some delay on some common processes in the network, like message transmission delay, processing delay, time out, etc. This behavior will result that at each instance of a simulation would produce different results. The packets exchanged between sender and receiver is of a fixed rate transmission $\lambda$ based on a Poisson distribution. Nodes that have packet queued are able to transmit it out using in each available bi-directional link channel. 
Our work extends the chance of contacting of the agents that arranged at the fixed cluster from nodes which are distributed randomly within the network. Agents navigating through the network for delivering messages must understand these clustered nodes whenever they are initiating a new route request and thereby increasing the degree of spatial coordination (agents must be on the same place). The temporal coordination has been enhanced with the introduction of a short waiting delay offered to each nodes/agents by the clustered overheads packets. This node - agent coordination will reduce the number of hops and waiting time in spite of further increase the overheads packets hang around with agents and head to highly the agent-chasing problem. The place hosted for the clustered agents can be called as the overlapped area within the network and the detainment period by the agents can be called agent periods.

This overlapped area actually offers a temporary space to be used by all agents for sharing network knowledge, and exchanging messages. Thus when an agent come a fresh it can exploit all other agents who are currently experiencing their agent periods. Here the traveling agents are allowed to carry the information of already visited clusters along with them. The idea behind this is to capture and share the partial network information present with roaming agents. The integration of all such partial information at a common overlapped area helps cooperative tasks like taking the decision for next destination, suitable exchange of messages between agents, getting up-todate knowledge of the network and reducing unnecessary redundant overhead packets used to visit nodes.

Thus the model of coordination clustered network area where the autonomous agents will be able to deliver messages within a large network with the cooperative communication between them at suitable overlapped area is necessary. There is need of knowing the routes proactively or reactively where part of the network capacity is used for exchanging chunks of routing table data.

We built network simulator to evaluate this proposed algorithm. The simulator supports physical, link and routing layers for single/multi hop ad-hoc networks. We assume that IEEE 802.11 Distributed Coordination Function (DCF) or MAC protocol which uses Channel Sense Multiple Access with Collision Avoidance (CSMA/CA) already deployed. Successfully received packet by receiver's interface is packet whose SNR is above a certain minimum value otherwise the packets cannot be distinguished from background noise/interference. Packets are transmitting through physical layer in accordance with Poisson distribution. Communication between two nodes in IEEE 802.11 uses TCP signaling before the actual data transmission takes place. Simulation simulates this with random hearing to link's condition. If link allow packets to be sent, then sender executes some packets already queued. To execute preferential event in sequentially distributed events, we used a simple approach that consists of applying a different time-event execution by means of the triggering event sequences action. The lower and upper bound of the queuing interval are set such that they do not interfere with predefined timers used by the other events for layers and modification events.

\section{B. Nodes}

Nodes are equipped with antenna module installed as capable of dynamically adjusting the transmission energy used to communicate with other nodes. Industrial standard of antenna module supports a management for controlling this energy consumption. The energy consumption required to transmit packet between nodes $\mathrm{A}$ and $\mathrm{B}$ is similar to that energy required between nodes $\mathrm{B}$ and $\mathrm{A}$ if and only if the distance and the size of packet are same. The coverage distance range of the nodes is a perfect symmetric unit disk (omnidirectional). If $\mathrm{d}_{\mathrm{x}, \mathrm{y}} \leq \mathrm{r}_{\mathrm{x}} \rightarrow \mathrm{x}$ and $\mathrm{y}$ can see each other. This assumption may be acceptable in the condition that interference in both directions is similar in space and time; which is not always the case. Usually interference-free Media Access Control (MAC) protocol such as Channel Sense Multiple Access (CSMA) may exist. In addition, wireless link channel is assumed to have no physical noise; i.e., the errors in packet reception due to fading and other external interferences are not considered as a serious problem. Packets from sender to receiver will be transmitted as long as the bandwidth capacity is sufficient and the received signal to noise ratio (SNR) is above a certain minimum value. Thus every packet successfully received is acknowledged at the link layer and deencapsulate at the higher layer. Each node is capable of measuring the received SNR by analyzing overheard packet. A constant bit error rate (BER) is defined for the whole network. Whenever a packet is going to be sent, a random number is generated and compared to the packet's CRC. If the random number is greater, the message is received, otherwise it is lost. The default value for the BER is 0 , which means there is no packet loss due to physical link error.

Energy is power kept in each node. Heinzelman et al. assumed that the radio dissipates $\mathrm{E}_{\text {elec }}=50 \mathrm{~nJ} / \mathrm{bit}$ to run the transmitter or receiver circuitry and $\varepsilon_{\mathrm{amp}}=100 \mathrm{pJ} / \mathrm{bit} / \mathrm{m}^{2}$ for the transmit amplifier [7]. The radio model is shown in the Figure 5 below.

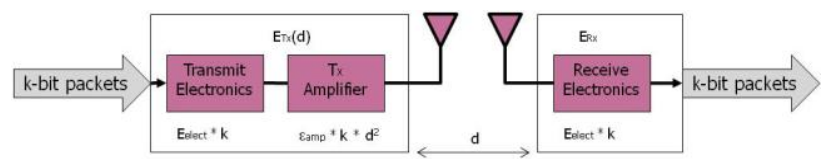

Figure 5. The radio model.

Thus, to transmit a k-bit message a distance $\mathrm{d}$ using this radio model, the radio expends:

$$
\begin{gathered}
E_{T x}(k, d)=E_{\text {Tx-elect }}(k)+E_{T x-a m p}(k, d) \\
E_{T x}(k, d)=E_{\text {elect }} * k+\epsilon_{\text {amp }} * k * d^{2}
\end{gathered}
$$

and to receive this message, the radio expends:

$$
\begin{gathered}
E_{R x}(k)=E_{R x-\text { elect }}(k) \\
E_{R x}(k)=E_{\text {elect }} * k
\end{gathered}
$$

Let $E_{\text {min_i }}$ is the minimum energy ratio of node $I$ at which a node can still receive, process, and transmit packets. Node $\mathrm{j}$ finds out the energy level of neighbor node I through analyzing of received reply packet from node $I$ as it responded the previous transmitted Hello packets. The computation of $E_{\min \_i}$ is done through two-step propagations. The use of two-steps 
propagation model is to simulate interactive propagation in the operation of the protocol in dynamic environment. As a future research, the appropriate propagation model that best matches to this environment should replace the simple two-steps model presented here [9][10]. The two-steps propagation model is appropriate for outdoor environments where a line of sight communication existed between the transmitter and receiver nodes and when the antennas are omni-directional. The twosteps propagation model assumes there are two main signal components. The first component is the signal traveling on the line of sight to reached neighbors along with its reply from neighbors and the second component is a confirmation packet transmitted to selected neighbors.

\section{Agents}

In this paper we introduce mobile agents to hop around the networks for delivering packets. The agents are allowed to meet with other agents at fixed overlapped places as has been mentioned. The cooperation among agents will mutually benefit each other by cooperating in delivering packets. In this current flexible and decentralized framework any autonomous node can send message to any other node at any instant within the network by just issuing a mobile agent. The agent then communicates with other agents to determine the proper one to carries the message to the corresponding destination node. The selected agent then becomes responsible for delivering the message to proper destination. Analog to the real life, these agents actually play the role of messengers and the selected agent play the role of post offices in the ad hoc wireless scenario. Such cooperation among agents scheme has been explicitly designed to reduce the agent traffic in the network. The unnecessary redundant node visits made by the agents to reach destination node has been avoided by sharing and merging with other agents. These agents take the responsibility of providing communication services and improvement of overall traffic in the network.

While delivering messages, an agent will maintain the path records of all the visited nodes in both clustered networks and its corresponding topology. Carrying this network information provide coordination and share the updated network knowledge with other agents. This information field carries topology tree along with numeric values of this membership list (of nodes) collected from each clustered network. In few cases the length of the information list carried by an agent gets longer due to the course of journey made from agent to reach the proper destination. If this happened, the list has been restricted using the hop count limit in order to avoid a huge series of data to be carried along by an agent and subsequent nodes. No packet is allowed to travel forward further whenever its hop count has got exhausted and it is compelled to move back to its originating source node/agent. Thus whenever an agent has finished its forward journey it will eventually follow the same path back to the source node.

The objective of the navigation procedure is to minimize the hops between the agent at overlapped location (current location of the node where the agent is residing) and the source and destination node's location. This criterion would enable an agent to select a neighbor of its current location and take out the packets to the destination nodes. If there is no neighbor available at that instant of time satisfying the above-mentioned criterion, the agent waits for a pre specified amount of time (randomly) and tries to communicate with other agents (any agents can be reached) to get its knowledge. Such contacted agents will respond the request. Through intensive communication among agents, the best agent can be selected to take responsibility for delivering received packets.

In the simulation, when a source node within the ad hoc network wants to send some packets, it immediately senses whether an agent is needed. Each such agent attaches with itself a topology bag to accommodate the request (certain) destination node. This bag is of a given capacity, which can be made full or can be made empty. The source node after initiating the agent puts the packets to (appropriate) agent. The agents are able to exchange these packets with other agents on having suitable coordination with them, which will have the best route path with minimum hops to reach destination node. These agents will be inactive automatically when there is no more packets need to be delivered.

Because of high degree of mobility, the topology will change and it is assumed that the agent will eventually succeed to migrate [2][11]. Whenever an agent wants to leave its current location for delivering packets to some unknown networks it will collect the topology list information from the nodes and will try to reach for a boundary node through which it may get an exit point. The node lying at the boundary will have neighbors from two or more different agents and can act as gateway nodes to other clustered network area. Thus if an agent can reach such a cluster area boundary, it can start visiting a fresh (other) agents. As the location of the agents can be made available from any node of that same clustered network area it can easily track the new nodes there, which has been compulsory. Though the order of cluster visits take place in a random manner still the redundancy in the path visit has been avoided by maintain the path visit list (using BFS function). The agents are free to roam among the overlapped area within the network in a random manner. This agents' capability will be presented on the next paper.

\section{PERformance EVAluation}

In this section, we evaluate the performance of the routing scheme for packets delivery within clustered ad hoc wireless network areas. We first describe our simulation implementation, performance metrics and methodology, and then we evaluate the agents initiated packets delivery scheme. The results confirm that the agents and their coordination routing algorithm at overlapped area are very efficient in delivering packets under high traffic. The result will also show that living agents in the system as there are packets spread around the clustered network areas; through suitable exchange and message sharing then the number of successful traffics get considerably increased with time.

We started to consider network composed of 60 nodes distributed into two area domain and 2 agents that are located within overlapped area between network areas. The network was set 400x200 areas, with overlapped region width of 20 . Fig.6 showed this view of simulation. The movement of the nodes is made random. Nodes are allowed to move at average speeds of $20 \mathrm{~m} / \mathrm{sec}$. Simulation is run for 100 cycles with 
movements' parameter was set random (i.e. direction, distance, and speed). Nodes are restricted to move only inside in its area. The following simulation with nodes' movement to other network area, including overlap area will be presented. In the subsequent simulations, the number of nodes is raised up along with combinations of different agents and various width of overlapped area.

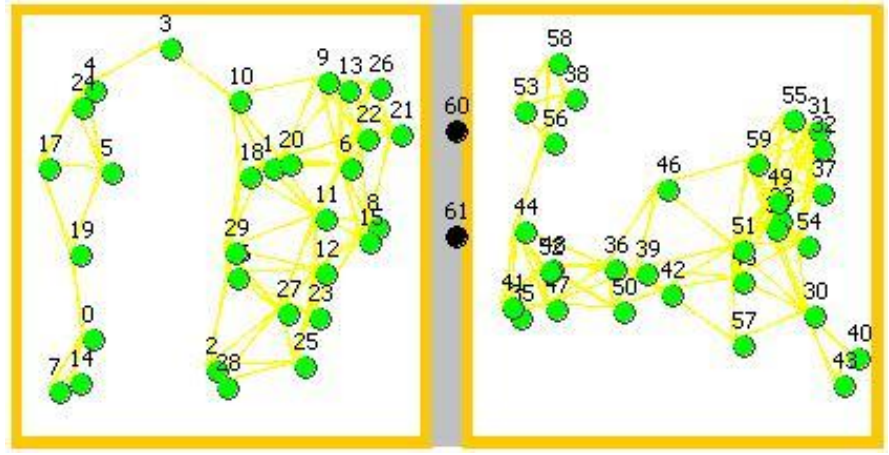

Figure 6. Initial network topology.

The performance of the agents' capability is evaluated using the following two criteria: i) Number of packets whose data contained in the propagation mechanism i.e. the total amount of packet traffic destined at agent to relay packets issued by the nodes in the network. ii) The time period selected as RTT period for successful packets delivery through network; both between source - agent and agent - destination. Network evaluation is intended to investigate the successful transmission rate in the hops between source and final destination nodes and then followed by several network performance analysis that are marked as scenario 1 , scenario 2 , scenario 3 , and scenario 4 . At each of scenario, network with similar initiator nodes are loaded with different packets length. We analyze these variables to determine their effects on the minimizing number of hops and average end-to-end delay for packets delivery. The simulation computes different hops/relay connectivity for the packets flow in the delivering packets from source to final destination.

As showed at Fig. 7(a), MANET tends to discover more successful data packets transmission in densely mobile environment (due to moving direction that getting closer to agents). It has been described in section III(b) and III(c), the network topology structure actually consists of two sub protocols, i.e. node topology protocol and agent topology protocol. In the case that many nodes located adjacently each other, thus every node periodically broadcasts Hello packets to denote its presence. Agents do the same sequences to adapt domain environments and to update network topologies for both of proximity areas. This extensive broadcast leads to unavoidable redundant packets for nodes located near overlapped zone. This is depicted in Fig.7(c) where high traffic is identified in the subsequent cycles. These agents, however, are intended to avoid global flooding and save network resources, but in such discovered case, agents are suffered in servicing network.

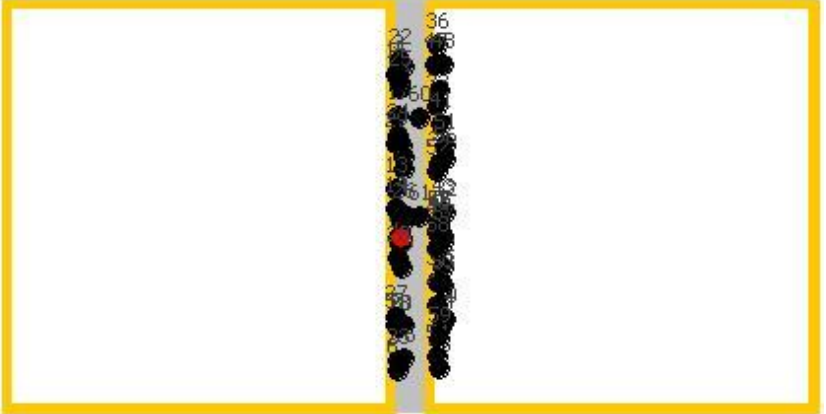

(a)

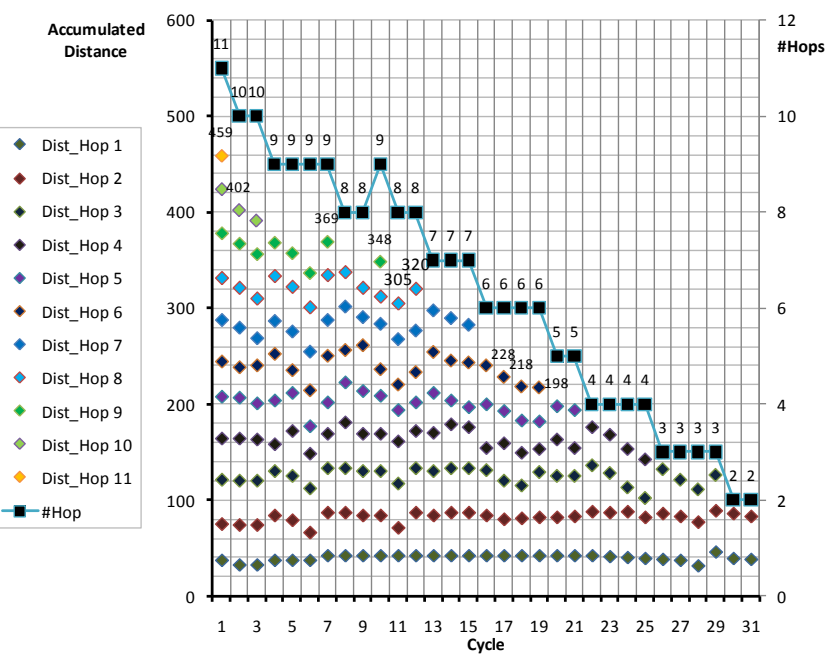

(b)

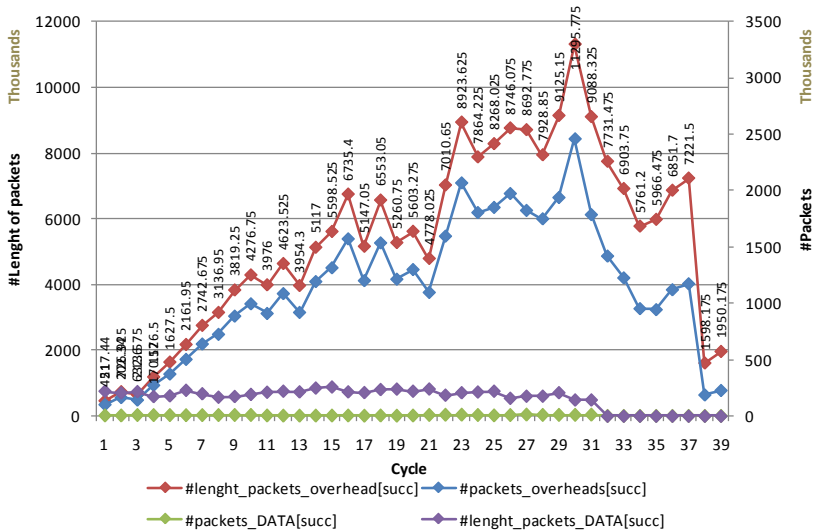

(c)

Figure 7. Simulation with nodes' movement direction staticly "closer to Agents" for network with 2 agents and 60 nodes in the two network areas. (a) Network topology at final cycle. (b) Accumulated hops and distances of successful data transmission for different cycle. (c) Broadcasted packets during simulation.

In the highly mobile environments (due to node mobility), more long-successful data packets delivery can be discovered. This is explained by the fact that when the nodes are highly mobile, paths are difficult to be maintained and hence far-away transmission tend to last for a very short amount of time since the probability for a path break is larger when nodes move faster. 
When nodes move slower these paths tend to be more stable and hence services tend to be available for a longer time. Such of this occurrence is explained in the Fig. 8.

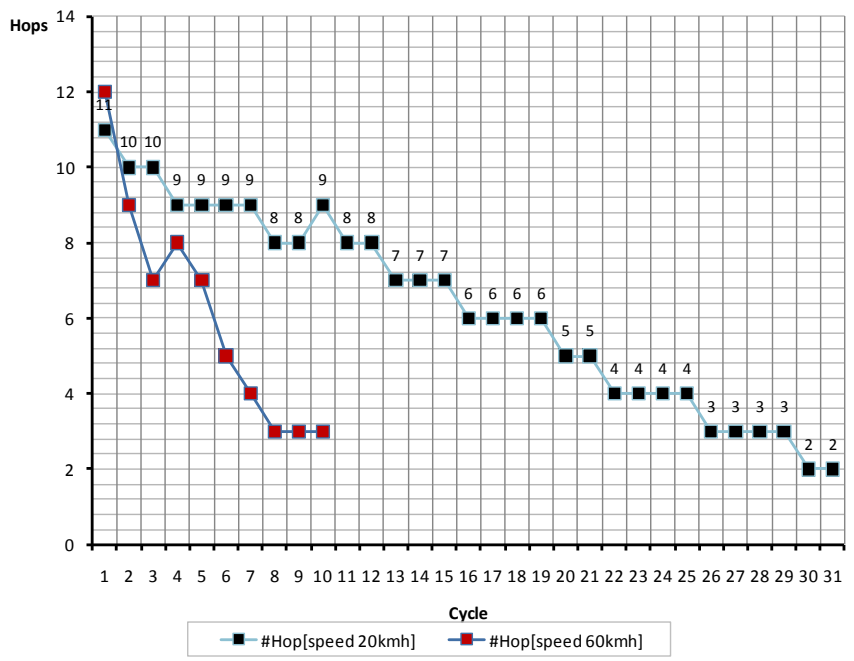

Figure 8. Hops of successful data transmission for different speed of simulation with movement direction "closer to Agents" for network with 2 agents and 60 nodes in the two network areas.

It is obvious from Fig. 7 that maximum successful data packets transmission can be achieved at low mobility when nodes are in overlapped area. The values of average successful data transmission over various mobility speeds are presented in Fig. 8. It is evident from this figure that the average successful data transmission actually decreases when speed increases and its direction are far from agents.

In order to evaluate the movement direction to network traffic, we put aware of the average duration of successful data packet transmission between a node and final destination through various movement directions. As previously predicted, the topology protocol would perform better in low mobility speed and direction of approaching agents. This is explained by the fact that the data transmission services discovered in that setting would be adequate for nodes to complete their transaction. The inverse would be true for low average successful data packet transmission duration, where a setting consists of high mobility speed and direction of away from agents is used for the topology protocol. Such situation can be concluded from Fig. 7, Fig. 8, and Fig. 9.

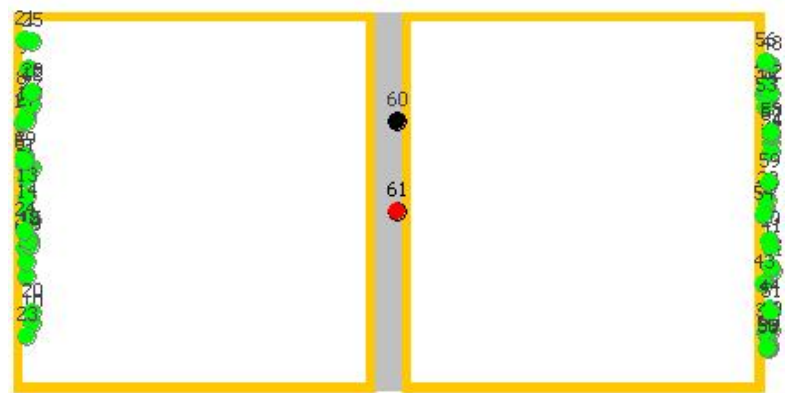

(a)

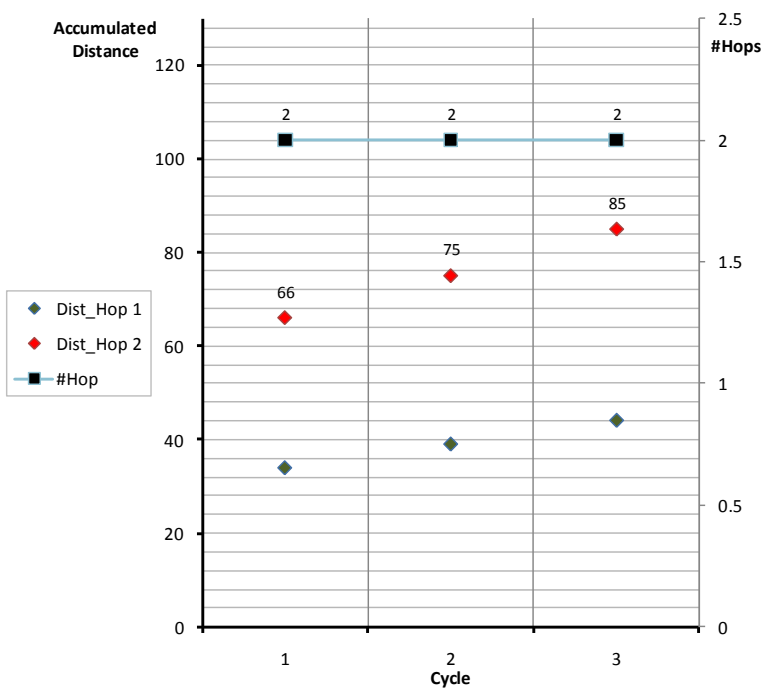

(b)

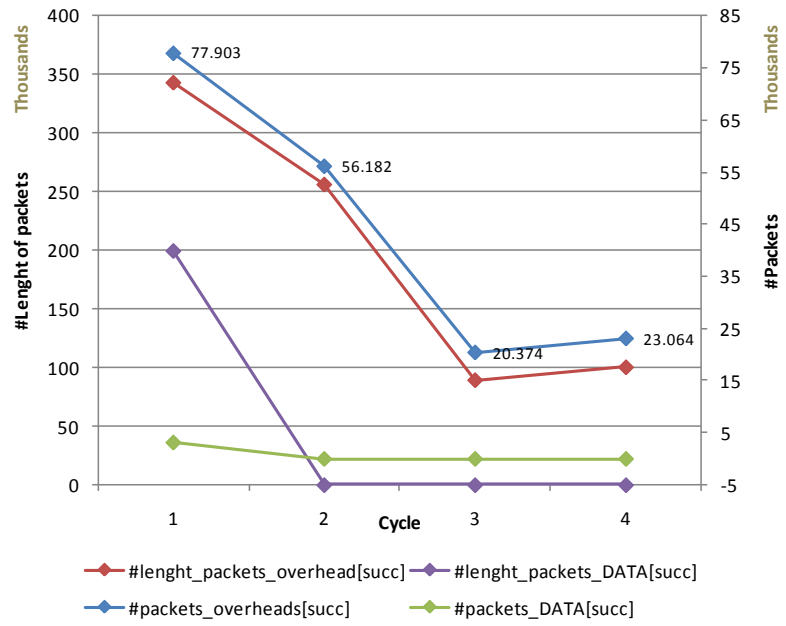

(c)

Figure 9. (a) Network topology at final cycle. (b) Accumulated hops and distances of successful data transmission for different cycle of simulation with movement direction "away to Agents" for network with 2 agents and 60 nodes in the two network areas. (c) Broadcasted packets during simulation.

Simulation was further modified in order to evaluate agents' service information in routing every relayed packet. Each node that is broadcasting this topology update packets sets the TTL (Time to Live) field in these packets equal so that they will be dropped at agents at overlapped area. Every node whose instance location is in overlapped area is set to as agents. Agents listen to information gathered from topology update packets, modify its table and then periodically broadcast its topology table back to nodes in the network. In the situation, where more nodes are located in the overlapped area, overhead traffic among agents leads to significantly higher overhead packets interaction (approximately $125 \%$ in average) and at the same time it fail to manage the transmission as no other nodes are discovered nor almost the nodes cannot reach agents. The following figures depict the behavior of network at different movement directions which is grouped as the final situation of network topology (in the (a)), the distance of successful data packets transmission at each hop (in the (b)), and the correlated broadcasted packets during simulation. 


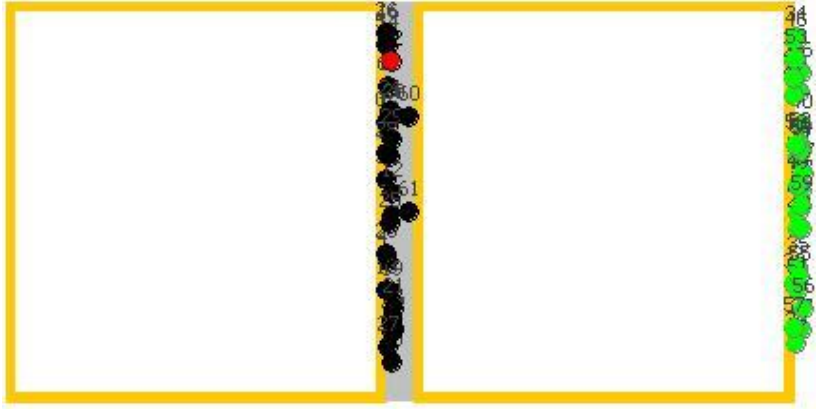

(a)

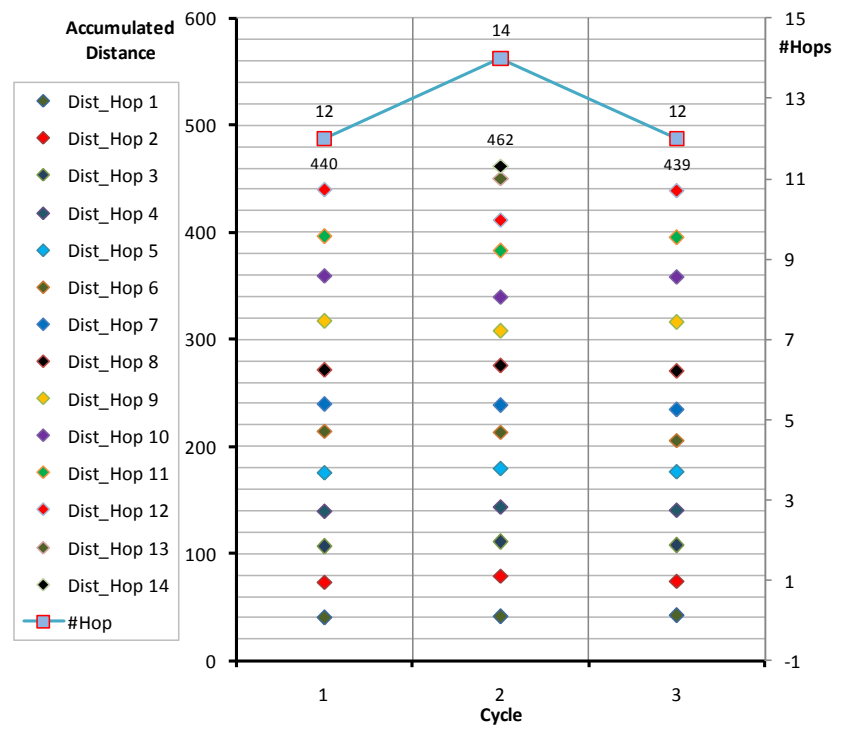

(b)

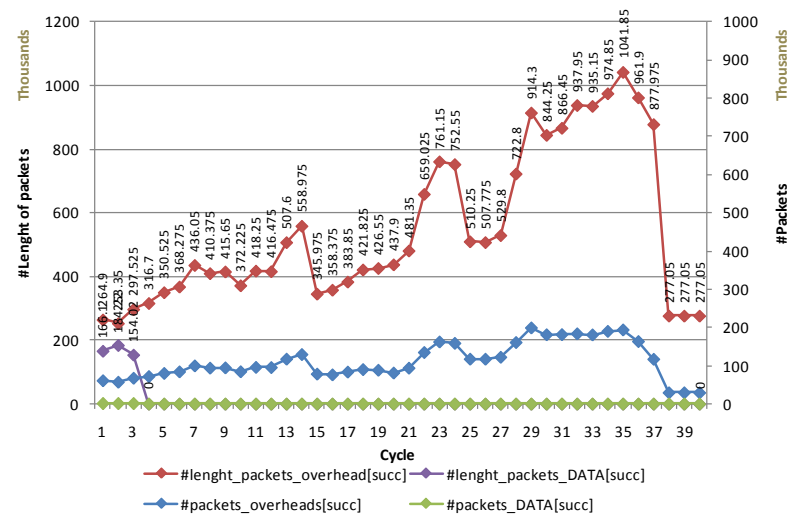

(c)

Figure 10. Simulation with nodes' movement direction staticly to East for network with 2 agents and 60 nodes in the two network areas. (a) Network topology at final cycle. (b) Accumulated hops and distances of successful data transmission for different cycle. (c) Broadcasted packets during simulation.

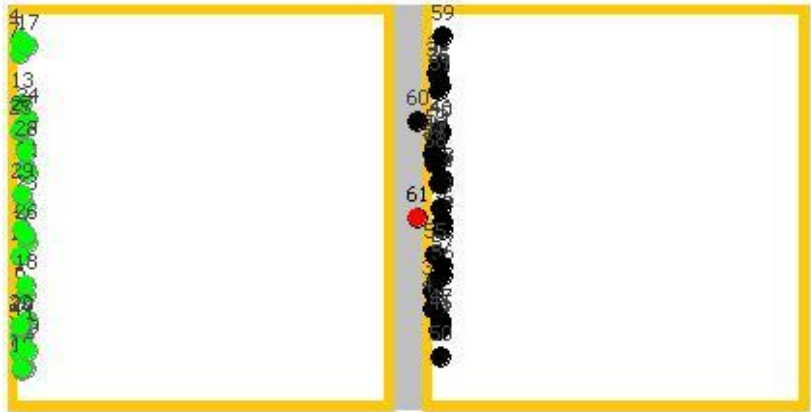

(a)

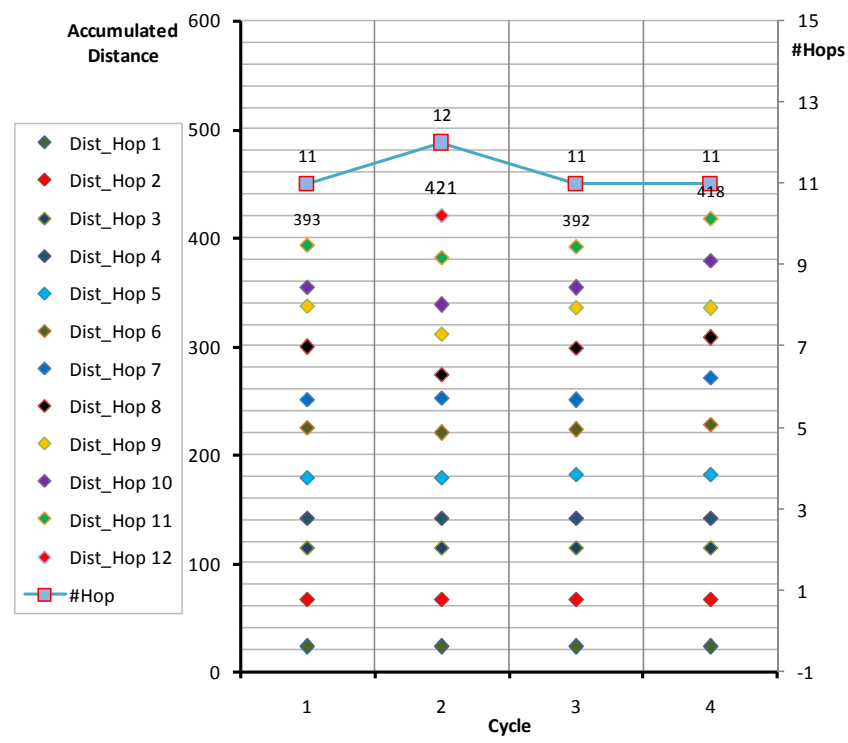

(b)

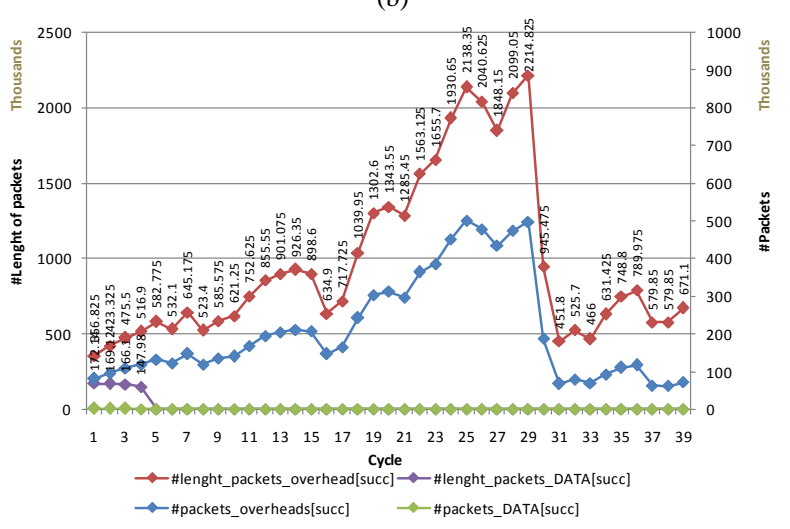

(c)

Figure 11. Simulation with nodes' movement direction staticly to West for network with 2 agents and 60 nodes in the two network areas. (a) Network topology at final cycle. (b) Accumulated hops and distances of successful data transmission for different cycle. (c) Broadcasted packets during simulation. 
In the Fig. 10(c) and 11(c), with the network movement direction is set to west and east, the broadcasted packets are significantly high at around cycle 28 to 30 . During those cycles, almost nodes in one area are located in the overlapped area and acted as agents. Communication among (more) agents and (less) nodes lead to flood the network with overhead packets. After such situation, the traffic tends to decline where nodes and agents are come to a stop at border and overlapped areas.

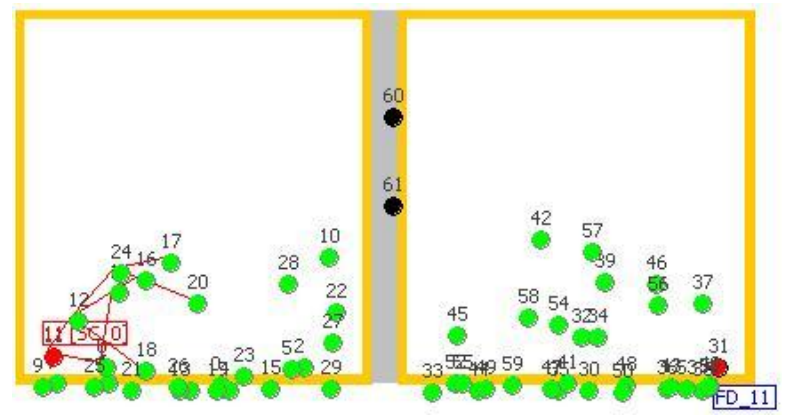

(a)

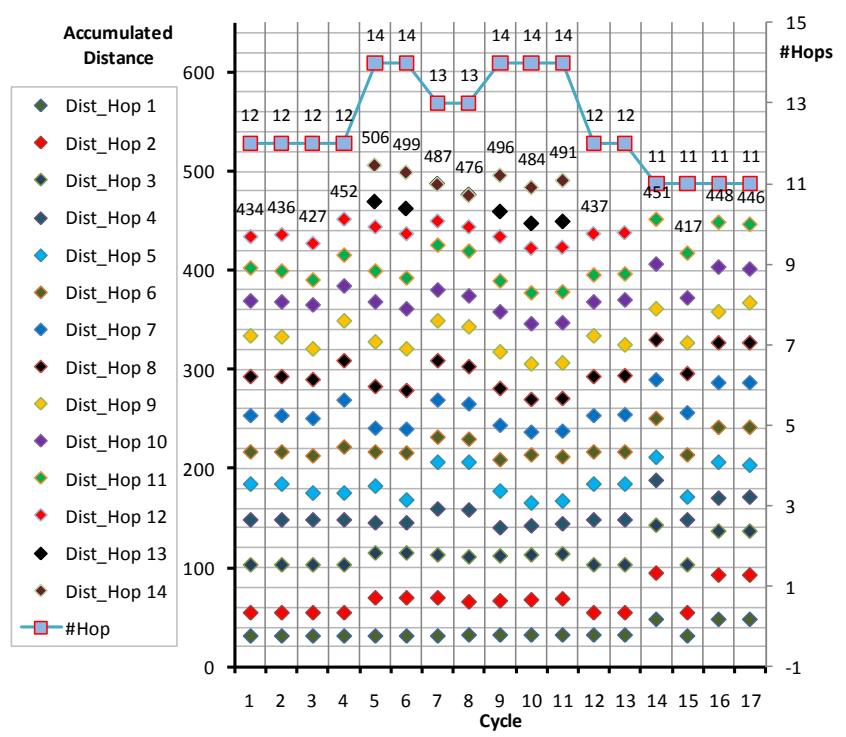

(b)

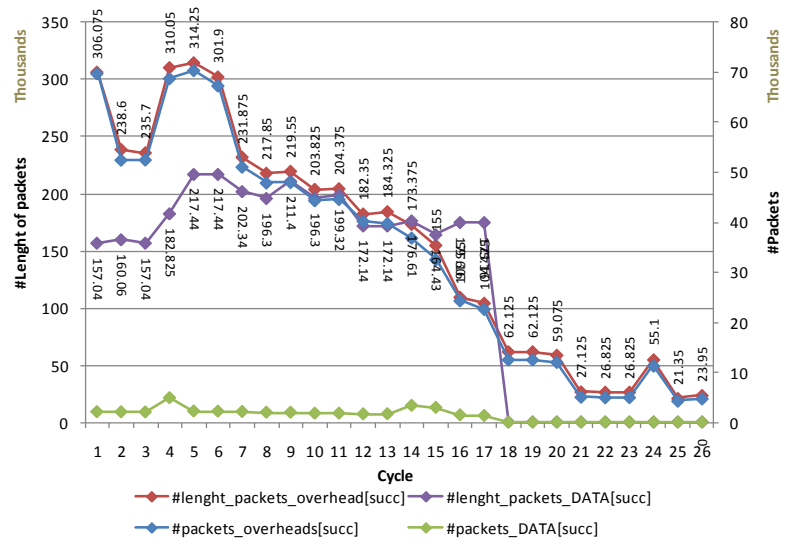

(c)

Figure 12. Simulation with nodes' movement direction staticly to South for network with 2 agents and 60 nodes in the two network areas. (a) Network topology at final cycle. (b) Accumulated hops and distances of successful data transmission for different cycle. (c) Broadcasted packets during simulation.
Subsequently, the scenario which every node has speed and direction that is determined of south and north is given below at Fig. 12(c) and the following Fig. 13(c). In all the two simulation models we did this simulation for 50 cycles with 60 nodes, as used previously for Fig. 10 and Fig. 11. Readings were taken for mobility of (speed) $20 \mathrm{~km} /$ hour. From the results it is evident that as the nodes coming near the border area increase; the traffic of both figures decline. Low packets nature suggests that more nodes stops looking for agents when no route path founded to reach them in the overlapped area. We simulated static agents in all figures.

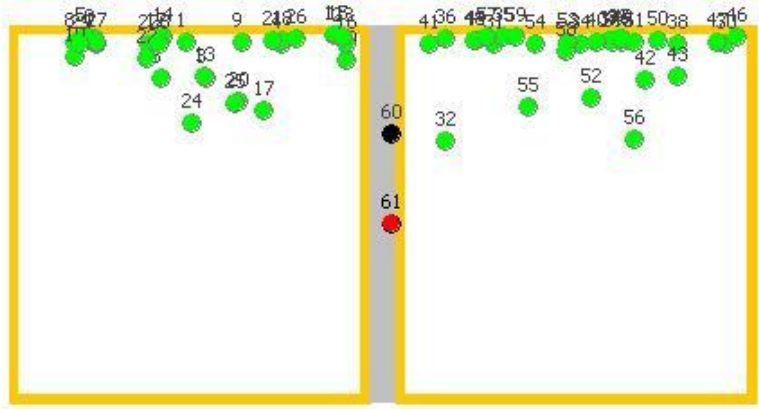

(a)

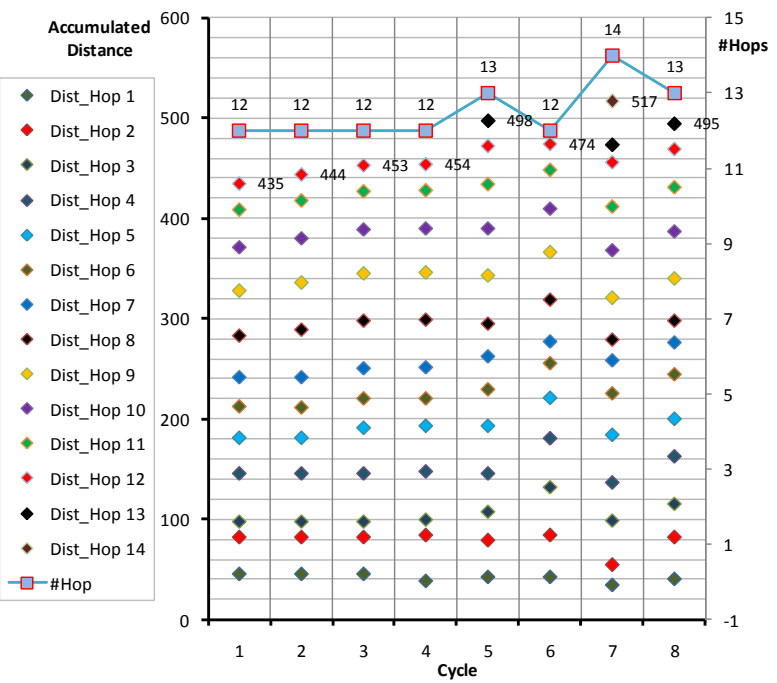

(b)

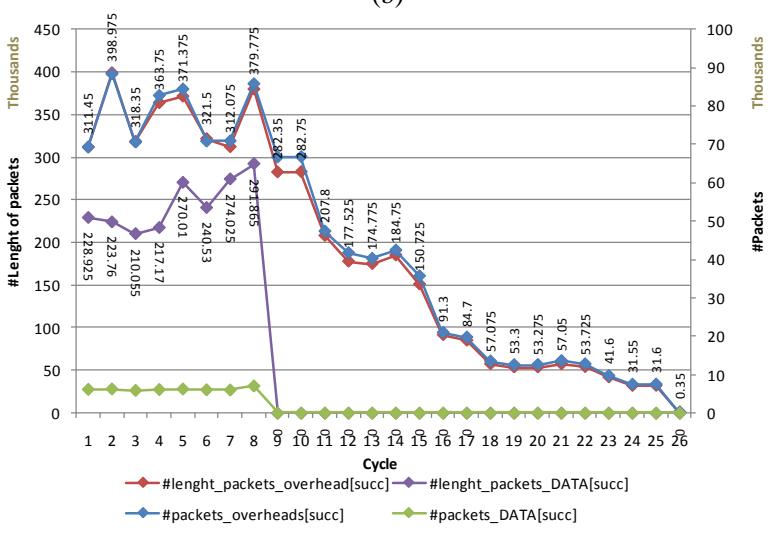

(c)

Figure 13. Simulation with nodes' movement direction staticly to North for network with 2 agents and 60 nodes in the two network areas. (a) Network topology at final cycle. (b) Accumulated hops and distances of successful data transmission for different cycle. (c) Broadcasted packets during simulation. 
Related to density, we simulated two scenarios. The first scenario included 120 nodes that are moving on two areas of total size $400 \times 400$ meters, following the random waypoint model with average speed $20 \mathrm{~m} / \mathrm{s}$ (minimum speed is still $0 \mathrm{~m} / \mathrm{s}$ ). The second scenario was identical to the previous one but involved only 60 nodes. Both scenarios had duration of 50 step movements each. The results are confirmed with previous scenarios. Different network density showed the same pattern of data packets transmission duration distributions, but the number of successful transmission in the sparsely scenarios is lower on the average of the others found in the first scenario. This is due to the fact that re-discoveries network topology is more frequent in a dense environment. Other fact is also obtained that overhead routing packets are marginally increased in size in order to encapsulate transmission information when density is increasing. The size of overhead packets plays a significant role under high-density cases where congestion is present. Hence, an increase in node density would lead to an increase in overhead packets and such as loss of data packets transmission due to congestion would be discovered.

\section{CONCLUSION}

In this paper, we evaluate the successful data packets delivery during packets transmission of MANETs with multihop routing. With network whose topology always changed all the time, then successful of data packets transmission is mainly depend on the situation of nodes to others. In the densely populated network, this is may not be the issues. On the contrary, if nodes stand apart from other and with their moving directions as such they cannot reach others, then no packets can be transmitted. In the simulation, agents have cascading effects. With their inherent advance capabilities, e.g. relay received packets, to examine the network topologies, determine the next destination of received packet, and guide the route path of packets transmission to reach the destination, agents are mainly to achieve optimum transmission while maintaining connectivity among areas. They can reduce the route of large number of unnecessary packets, particularly in the transmission of packets destined to other area. Except these excellent capability, communication among agents must be take into consideration. Node's movement direction, as key connectivity of nodes to form MANET topology, is evaluated to show the importance of agents. In the dynamic mobile environment, since there are more alternative paths for agents through which node can reach agents and also more alternative agents available in the overlapped area, hence a failure of one or more paths doesn't necessarily mean that the node cannot access the agents in order to deliver packets to reach final destination. Simulation results presented in section IV show that this is not always true, particularly, when nodes are moving away from agents. In such situation, the density increases, despite the existence of multiple paths and agents, the average successful data packets transmission is decreased. This is explained by the fact that pair of source and final destination nodes failed to maintain its connectivity as its distance getting out of agents' proximity. On the contrary, more nodes whose moving direction is getting closer to agents will create more contention for accessing the channel and transmitting service advertisements. Hence, more packet collisions occur and large energy is consumed. The total number of successful data packets transmission however is higher in dense environment with this movement direction. This means that high density may increase the number of successful data packets delivery but it decreases their quality in terms of availability. In nearly future work, we plan to add agents' capabilities to cover the nodes which move through each network areas. Another further investigation need to be conducted on dynamic routing advantages and factors which affect routing mode, e.g., flow type, delay, throughput/delay/reliability tradeoffs between wireless network areas, etc.

\section{ACKNOWLEDGMENT}

The authors would like to thank the anonymous reviewers for the helpful comments and suggestions.

\section{REFERENCES}

[1] Christopher N. Ververidis and George C. Polyzos, "Impact of Node Mobility and Network Density on Service Availability in MANETs", Proc. 5th IST Mobile Summit, Dresden, German, June 2005

[2] Romit RoyChoudhury, Krishna Paul, and S. Bandyopadhyay, "MARP: A Multi-Agent Routing Protocol for Mobile Wireless Ad Hoc Networks" Autonomous Agents and Multi-Agent Systems (Kluwer), 8 (1): 47-68.

[3] Galli et al., "A Novel Approach to OSPF-Area Design for Large Wireless Ad-Hoc Networks", IEEE ICC'05, Seoul, Korea, May 16-20, 2005

[4] Thomas R.H., Phillip A. S., Guangyu P., "Evaluation of OSPF MANET Extensions", Boeing Technical Report: D950-10897-1, The Boeing Company, Seattle, July 21, 2005

[5] Henderson, T., et al., "Evaluation of OSPF MANETExtensions", Boeing Technical Report D950-10915-1, March 3, 2006,

[6] Spagnolo, P., "Comparison of Proposed OSPF MANETExtensions", The Boeing Company, October 23, 2006

[7] Heinzelman, W., Chandrakasan, A., Balakrishnan, H. "Energy-efficient communication protocol for wireless microsensor networks". In: Proceedings of the 33rd International Conference on System Sciences (HICSS): 1-10, 2000.

[8] M. S. Corson, S. Batsell, and J. Macker, "Architecture consideration for mobile mesh networking", Proceedings of the IEEE Military Communications Conference (MILCOM), vol. 1, pp. 225-229, 21-24 Oct. 1996.

[9] Chang-Woo Ahn, Sang-Hwa Chung, Tae-Hun Kim, and Su-Young Kang. "A Node-Disjoint Multipath Routing Protocol Based on AODV in Mobile Adhoc Networks". Proceeding of Seventh International Conference of Information Technology ITNG2010, pp. 828-833, April 2010.

[10] Zheniqiang Ye, Strikanth V. Krishnamurthy and Satish K. Tripathi, “A Framework for Reliable Routing in Mobile Ad HocNetworks", IEEE INFOCOM, 2003.

[11] Romit RoyChouldhury, S. Bandyopadhyay and Krishna Paul, "A Mobile Agent Based Mechanism to Discover Geographical Positions of Nodes in Ad Hoc Wireless Networks", Accepted in the 6th Asia-Pacific Conference on Communications (APCC2000), Seoul, Korea, 30 Oct. - 2 Nov. 2000.

[12] M. Nidd, "Service Discovery in DEAPspace", IEEE Personal Communications, August 2001, pp. 39-45.

[13] G. Schiele, C. Becker and K. Rothermel, "Energy-Efficient Clusterbased Service Discovery for Ubiquitous Computing," Proc. ACM SIGOPS European Workshop, Leuven, Belgium, Sept. 2004.

[14] N. H. Vaidya, "Mobile Ad hoc networks routing, mac and transport issues", Proceedings of the IEEE International Conference on Computer Communication INFOCOM, 2004.

[15] Cisco, OSPF Design Guide, Document ID: 7039, Aug 10, 2005.

[16] Indukuri, R. K. R. (2011). Dominating Sets and Spanning Tree based Clustering Algorithms for Mobile Ad hoc Networks. IJACSA - 
International Journal of Advanced Computer Science and Applications, 2(2). Retrieved from http://ijacsa.thesai.org.

[17] Division, I. S. (2011). A Novel approach for Implementing Security over Vehicular Ad hoc network using Signcryption through Network Grid. IJACSA - International Journal of Advanced Computer Science and Applications, 2(4), 44-48.

Kohei Arai

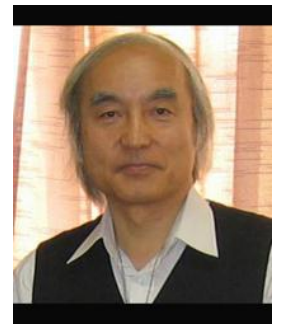

\section{AUTHORS PROFILE}

Prof K. Arai was born in Tokyo, Japan in 1949. Prof K. Arai's major research concern is in the field of human computer interaction, computer vision, optimization theory, pattern recognition, image understanding, modeling and simulation, radiative transfer and remote sensing. Education background:

- $\quad$ BS degree in Electronics Engineering from Nihon University Japan, in March 1972,

- $\quad$ MS degree in Electronics Engineering from Nihon University Japan, in March 1974, and

- $\mathrm{PhD}$ degree in Information Science from Nihon University Japan, in June 1982.

He is now Professor at Department of Information Science of Saga University, Adjunct Prof. of the University of Arizona, USA since 1998 and also Vice Chairman of the Commission of ICSU/COSPAR since 2008. Some of his publications are Routing Protocol Based on Minimizing Throughput for
Virtual Private Network among Earth Observation Satellite Data Distribution Centers (together with $\mathrm{H}$. Etoh, Journal of Photogrammetory and Remote Sensing Society of Japan, Vol.38, No.1, 11-16, Jan.1998) and The Protocol for Inter-operable for Earth Observation Data Retrievals (together with S.Sobue and O.Ochiai, Journal of Information Processing Society of Japan, Vol.39, No.3, 222-228, Mar.1998).

Prof Arai is a member of Remote Sensing Society of Japan, Japanese Society of Information Processing, etc. He was awarded with, i.e. Kajii Prize from Nihon Telephone and Telegram Public Corporation in 1970, Excellent Paper Award from the Remote Sensing Society of Japan in 1999, and Excellent presentation award from the Visualization Society of Japan in 2009.

\section{Lipur Sugiyanta}

Lipur Sugiyanta was born in Indonesia at December 29, 1976. Major field of research is computer network, routing protocol, and information security. Education background:

- Bachelor degree in Electrical Engineering from Gadjah Mada University

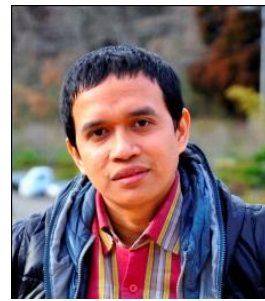
of Indonesia, in February 2000

- $\quad$ Magister in Computer Science from University of Indonesia, in August 2003.

$\mathrm{He}$ is now lecturer in Jakarta State University in Indonesia. Since 2008, he has been taking part as a $\mathrm{PhD}$ student in Saga University Japan under supervision of Prof K. Arai. 